\title{
The effect of an irrelevant directional cue on choice reaction time: Duration of the phenomenon and its relation to stages of processing
}

\author{
J. RICHARD SIMON, ENRIQUE ACOSTA, JR., STEVEN P. MEWALDT, and CYNTHIA R. SPEIDEL \\ The University of Iowa, Iowa City, Iowa 52242
}

\begin{abstract}
Two experimental paradigms were used to investigate the interference which occurs in a choice RT task when the source of a stimulus does not correspond with its meaning. In one paradigm, subjects were instructed to delay executing their response to a red or green light until they heard a go signal which was presented $0,150,250$, or 350 msec after the light. In the other paradigm, subjects translated the pitch of a tone into an appropriate keypress response. For some groups, the stimulus response connections were specified prior to or simultaneously with the presentation of the stimulus tone, while, for other groups, the labeling of the response keys followed the onset of the tone. Results indicated that irrelevant directional cues produce interference which affects the response selection stage and persists for approximately 250 msec.
\end{abstract}

In a series of experiments, Simon and his associates have shown that certain auditory and visual displays provide irrelevant directional cues which affect the processing of relevant information in the display. For example, responses to monaural or monocular commands of "left" or "right" are significantly slower when the symbolic content of the command does not correspond with the ear or eye stimulated than when it does correspond. The irrelevant cue is not the ear or eye stimulated per se but, rather, a directional cue associated with the spatial location of the stimulus (Craft \& Simon, 1970; Simon, Small, Ziglar, \& Craft, 1970). Apparently, there is an initial tendency to react to the source of the stimulus rather than to its meaning and the necessity of overriding this inappropriate response tendency results in the slower information processing observed when the source and the content of a stimulus do not correspond. While there is some evidence that the irrelevant directional cue may facilitate as well as interfere with information processing (Simon \& Craft, 1970; Wallace, 1971). in this paper we will use the term "interference effect" as a shorthand way of referring to this stereotypic tendency to respond toward the stimulus source.

Reactions toward the source of stimulation have also been observed in tasks where the relevant and irrelevant cues involve different sensory modalities (Simon \& Craft, 1970) and even in tasks where the irrelevant cue precedes the onset of the relevant stimulus (Simon, Acosta, \& Mewaldt, 1975). A variety of other results can be explained in terms of an initial tendency to react toward the stimulus source; e.g., observed differences in reaction time (RT) to the onset and offset of lights and tones (Simon, Craft, \& Webster, 1971) and RT differences in an oddity task (Simon \& Craft, 1972). We have emphasized earlier (Simon, Craft, \& Webster, 1973) that the present phenomenon bears only the most superficial resemblance to the well-known Stroop effect (Jensen \& Rohwer. 1966). The purpose of the present research was to chart the time course of this potent phenomenon and to localize its effect in relation to the stages of information processing.

The procedure followed in Experiment I involved a modification of a task employed by Craft and Simon (1970) in which a red or green light, signaling a left- or right-hand response, was presented to either the left or right half of the visual field. In contrast to the Craft and Simon procedure where RT was measured from the onset of the light, the subjects in Experiment I were told to delay their response to the light until they heard a go signal, and RT was measured from the onset of this go signal. On some trials, the light and go signal occurred simultaneously. The RT on these trials, then, reflected the full extent of the interference which occurs when source and content of a command do not correspond. On other trials, there was an interval between the light and the go signal. We reasoned that subjects would use this interval to override the initial inappropriate tendency to respond to the source of stimulation. If the interval were "long enough," the subject would be able to complete the process of separating the irrelevant directional cue from the relevant symbolic cue, and there would be no interference reflected in his RT to the go signal. By gradually lengthening the interval between light and go signal, it should be possible to specify the point at which the interference effect no longer occurs. The length of this interval, then, provides an estimate of the duration of the interference effect.

Estimating the duration of the interference phenomenon was also one of the purposes of Experiment II. Another important purpose, however, was to determine whether the interference would 
occur at all in a task where the subject could not program the stimulus-response associations prior to stimulus presentation. The subjects pressed either a red key or a green key depending on the pitch of a monaural or binaural tonal command. Several variations of this basic task were employed. In some, the red and green keys were designated (i.e., "labeled") prior to the onset of the tone; e.g., the right key might be red and the left key green. Thus, upon onset of the tone, the subject had all of the information he needed to initiate his response. In other variations, the keys were not labeled until after the tone had been presented, and so the subject had to delay his response until he determined which key was red and which was green on that particular trial. Following a rationale similar to that of Experiment I, various intervals between tone onset and response-key labeling were investigated. We reasoned that any initial tendency to react to the source of the tone would tend to dissipate during the enforced delay before responding. By identifying the critical interval at which interference no longer occurred, we would have an estimate of the duration of the interference effect.

\section{EXPERIMENT I}

\section{Method}

Subjects. The subjects were 20 male and 20 female students enrolled in an introductory psychology course at The University of Iowa. All subjects were right-handed.

Apparatus. A Realist electric stereo viewer. Model 2062, was modified to provide the display. The modification involved removing the lenses and inserting an opaque partition which divided the visual field in half so that the left half was visible to the subject's left eye and the right half was visible to his right eye. In the center of each half of the field, red and green light-emitting diodes (LED) were mounted in translucent Plexiglas, with the green LED $1.5 \mathrm{~cm}$ above the red. These LED, which served as stimulus lights, were $0.5 \mathrm{~cm}$ in diam and installed $4.8 \mathrm{~cm}$ from the eye openings of the viewer; thus the visual angle subtended by each light was approximately $6 \mathrm{deg}$. A white warning light. installed $2.0 \mathrm{~cm}$ behind the translucent Plexiglas, was visible to both eyes. The viewer was mounted on a post and was adjustable along the vertical axis to each subject's eye level.

The go signal was a $100-\mathrm{Hz}$ tone at 94-dB SPL (re .0002 microbar) generated by a Hewlett Packard audio oscillator Model $200 \mathrm{AB}$ and presented to the subject binaurally through Permoflux PDF 8 300 -ohm impedance earphones. Two telegraph keys were positioned $30 \mathrm{~cm}$ apart on a table in front of the subject. The subject operated these keys with his right and left index fingers. The sequence of events was controlled by a BRS Foringer digital logic system and paper-tape reader. Reaction time was measured by a multivibrator clock accurate to the nearest millisecond. Timing began when the go signal was presented and stopped when a response key was pressed. The subject's response also terminated the stimulus light and the go signal. Responses, i.e., the key used, and RTs were recorded automatically on paper tape.

Design. The experimental design involved three within-subject factors and two between-subject factors. The within-subject factors were: command (red light or green light signaling a left or right response). location of the command (left or right visual field), and delay of the go signal $(0.150 .250$, or $350 \mathrm{msec})$. The between-subject factors were sex and color-response instructions. Half the subjects were instructed to press the right key for the green light and the left key for the red light, while the other ho'f were given the opposite color-response instructions. There were, then, four between-subject combinations to which 40 subjects were assigned, 10 to each group.

Procedure. Each subject performed on two identical blocks of 144 test trials. The blocks were separated by a short rest period. The sequence of trials within a block was constructed so that each of the 16 treatment combinations (i.e., 2 commands by 2 locations by 4 delays) occurred equally often in each third of the block, and no treatment combination repeated itself immediately. Each block was preced ed by a differ ent sequence of 16 warm-up trials. Subjects were also given 30 practice trials prior to the first block. Neither the practice nor the warm-up trials were included in the data analyses.

The sequence of events on each trial was as follows: Two seconds after the response on the preceding trial, the warning light flashed for $100 \mathrm{msec}$. One second later, either a red or a green light came on indicating which key to press. Then $0,150,250$, or $350 \mathrm{msec}$ later, the go signal was heard which cued the subject to initiate his response. A response made before the go signal was presented was not accepted by the apparatus, and the subject had to respond a second time for it to be recorded. These responses were recorded on a counter and occurred on less than $1 \%$ of the trials.

\section{Results}

Median RTs were computed for each subject for each of the 16 treatment conditions, i.e.. all combinations of the two commands, two locations of each command, and the four delay intervals. Trials involving incorrect responses or premature responses (defined as RTs of less than $100 \mathrm{msec}$ ) were not included in the analysis. These constituted $4 \%$ of the trials.

Figure 1 shows the means of the individual median

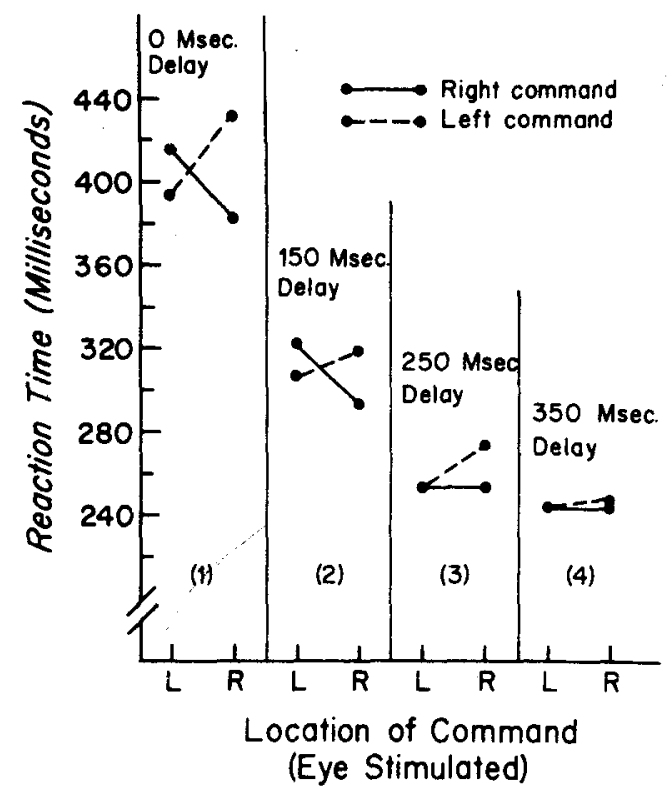

Figure 1. Effect of an irrelevant directional cue in a choice reaction time task involving a go signal. The basic task involved pressing either a left- or a right-hand key, depending on the color of a red or a green command light. The light appeared in either the left or the right half of the visual field, thus providing an irrelevant directional cue. The subject was instructed to delay executing his response until he heard an auditory go signal, which was presented either simultaneously with the command light (Panel 1) or following the light by 150, 250, or 350 msec (Panels 2 through 4). Reaction time was measured from the onset of the go signal. 
RTs for each condition. An overall analysis of variance revealed a significant main effect of delay interval, $F(3,117)=489.59, p<.001$. Note that $R T$ decreased as the interval between the command light and the go signal increased. There was also a significant main effect of command, $F(1,39)=7.69$, $p<.01$, reflecting the fact that subjects were faster in executing responses with their right (dominant) hands. The overall analysis also revealed a significant Command by Command Location interaction, $F(1,39)$ $=70.30, \mathrm{p}<.001$, and a Command Location by Delay Interval interaction, $F(3,117)=3.99, \mathrm{p}<.05$. Of major interest, however, was the significant Command by Command Location by Delay Interval interaction, $F(3,117)=19.04, p<.001$. Since the Command by Command Location interaction at each delay interval was of primary concern in the present experiment, separate analyses were conducted for each delay interval. These analyses revealed a significant Command by Command Location interaction $(\mathrm{p}<.01)$ at 0,150 , and $250 \mathrm{msec}$, but, obviously, no effects were significant at the $350-\mathrm{msec}$ delay. Figure 1 shows that, at each of the three shortest delay intervals, RT to the left command was faster when the light was located on the left side of the display than when it was located on the right. Similarly, at 0 and $150 \mathrm{msec}$, RT to the right command was faster when the light was on the right side than when it was on the left. This effect did not occur at the 250-msec delay interval.

\section{Discussion}

The paradigm followed in Experiment I (i.e., command light followed by go signal) permitted subjects to begin stimulus identification and response selection as soon as they saw the light. Lengthening the interval between the light and go signal allowed more time for these earlier stages of processing to proceed and resulted in faster R'Ts to the go signal. Clearly, however, subjects did not use the interval available between light and go signal to its full potential. Note, for example, that RT on the $150-\mathrm{msec}$ delay trials was only about $100 \mathrm{msec}$ faster than on the 0-msec delay trials. Our data also indicate that lengthening of the delay interval beyond about $250 \mathrm{msec}$ did not produce much additional decrement in overall RT. This latter finding suggests that the stimulus identification and response selection stages are essentially complete after about $250 \mathrm{msec}$.

Results of Experiment I indicate that $250 \mathrm{msec}$ is the approximate duration of the interference phenomenon under investigation. With a delay interval of $150 \mathrm{msec}$ between stimulus and go signal, both left and right responses were clearly slower when the source and the content of the stimulus did not correspond. When the delay interval was increased to $250 \mathrm{msec}$, the interference effect was significant only for the left response. When the delay interval was further increased to $350 \mathrm{msec}$, the source of the stimulus no longer had any detectable effect on RT. These results suggest that the effect of the irrelevant directional cue is localized in the latter stages of information processing, perhaps in the response selection stage. ${ }^{1}$ The traditional spatial compatibility manipulation (Fitts \& Seeger, 1953) would seem to involve the response selection stage since $R T$ varies depending on the response which is paired with a particular stimulus. In the classic compatibility task, however, stimulus location provides the relevant cue, whereas, in the present task, location provides an irrelevant cue. The locus of the effect of this irrelevant cue is certainly not obvious. Presumably, the response selection stage is involved, but other processing stages might also be affected. Experiment II provides additional evidence concerning the locus of the effect under investigation.

Our problem with the paradigm used in Experiment I was that it did not provide a foolproof means of separating stages of processing. There was no way of absolutely precluding subjects from beginning the response execution stage before they heard the go signal. We know that subjects did not ignore the go signal, for if they had, then premature responses or impossibly short RTs would have been recorded on virtually all of the long-delay trials, and this was clearly not the case. Still, the procedure used left something to be desired. In Experiment II, we continued to explore the duration of the interference phenomenon and, in the process, developed a new procedure for separating the response selection stage from the preceding stages of information processing. The procedure involved delaying the designation of the response keys in a choice RT task until after the stimulus had been presented. In this way, subjects were prevented from beginning the response selection stage until the instant specified by the experimenter.

\section{EXPERIMENT II}

\section{Method}

Subjects. The subjects were 24 male and 24 female students enrolled in an introductory psychology course at The University of Iowa. All subjects were right-handed and reported normal or corrected-to-normal vision and hearing.

Apparatus. The apparatus provided a measure of choice RT in a task which required the subject to press either a left- or a right-hand key labeled "red" or "green" in response to the pitch of a stimulus tone. The rule for translating pitch (high or low) to response (red or green key) remained constant throughout the experiment for a given subject. Two telegraph keys were mounted $8 \mathrm{~cm}$ apart on a table in front of the subject. One centimeter behind the keys was a panel containing two LEDs (Monsanto MV5491), each of which subtended a visual angle of approximately $36 \mathrm{~min}$. These diodes, each capable of producing a red or a green light, were located $1 \mathrm{~cm}$ above each telegraph key and were used to label the keys, one as the "red key" and the other as the "green key." By employing the LEDs, it was possible to vary the designation of the response keys from trial to trial, to delay labeling the response keys until after the stimulus tone was presented, and to manipulate the interval between stimulus 
presentation and labeling of the keys. A white warning light, used to signal the start of each trial, was installed in the center of the panel $7 \mathrm{~cm}$ above the LEDs.

Two Hewlett Packard Model $200 \mathrm{AB}$ audio oscillators were used to generate $100-\mathrm{msec}$-duration tones of 250 and $625 \mathrm{~Hz}$. The tones were presented to the subject either monaurally or binaurally through Grason-Stadler TDH-39 earphones. On monaural trials, the output level was $100 \mathrm{~dB}$ SPL (re .0002 microbar), while on binaural trials, the output level was decreased to $97 \mathrm{~dB} \mathrm{SPL}$ so as to yield approximately the same loudness as on the monaural trials.

Overall control of the sequence of events and data acquisition was achieved with the same equipment employed in Experiment I. Reaction time was measured from the instant the subject received sufficient information to press the correct key. Specifically, in those treatment conditions where the keys were labeled prior to the presentation of the tone, RT was measured from the onset of the tone. In those treatment conditions where the keys were labeled after the presentation of the tone. RT was measured from the instant the keys were labeled.

Procedure and Experimental design. Each subject performed on two blocks of trials, each block consisting of 24 warm-up trials and 144 test trials. The sequence of test trials was arranged so that each treatment combination occurred equally often in each set of 48 trials. The subjects received 12 practice trials prior to the first block, and there was a brief rest interval between blocks. Each trial consisted of a high-or low-pitched tone presented to the left ear, the right ear, or binaurally. The subjects' task was to translate the pitch information into a color-coded response; i.e.. they were told to press the red key when they heard one tone and the green key when they heard the other tone. The basic sequence of events consisted of a $100-\mathrm{msec}$ warning light at the start of each trial followed $1 \mathrm{sec}$ later by a monaural or binaural tonal command. Two seconds after the subject made a response, the warning light for the next trial was presented.

Eight subjects. four males and four females, were randomly assigned to each of the six treatment groups. Each group performed on a different variation of the basic task. Half of the males and half of the females in each group were instructed to press the red key in response to the high-pitched tone and the green key in response to the low-pitched tone, while the other half followed the opposite tone-color rule. For Group 1, the red and green keys were designated before the start of the experimental session and these labels remained fixed throughout the session. For half the subjects in Group 1, the left key was red and the right key.was green, while. for the other half, the reverse labeling was used. For Group 2, the keys were labeled $1 \mathrm{sec}$ prior to the presentation of the tonal commands (simultaneously with the onset of the warning light) and the labels varied from trial to trial; i.e., on one trial, the right key might be red and the left key green while on the next trial the reverse labeling might occur. For both Groups 1 and 2. the LEDs which labeled the keys came on simultaneously with the warning light and were turned off by the subject's response. For Groups 3, 4, 5, and 6, the keys were labeled $0,150,250$, or $350 \mathrm{msec}$, respectively, after the onset of the tone. As in the first two groups, the subject's response turned off the LEDs.

\section{Results}

Median RTs were computed for each subject for each of the six treatment combinations, i.e.. two commands (left or right) and three tone locations (left ear. right ear, or binaural). Trials involving errors $(2.3 \%$ of all trials) were not included in the computation of these medians.

Our primary concern was to examine the effect of irrelevant directional cues on the different treatment groups. Since directional cues were present only on the monaural trials, the major analysis was restricted to these trials. Figure 2 displays the means of the individual median RTs for the monaural treatment

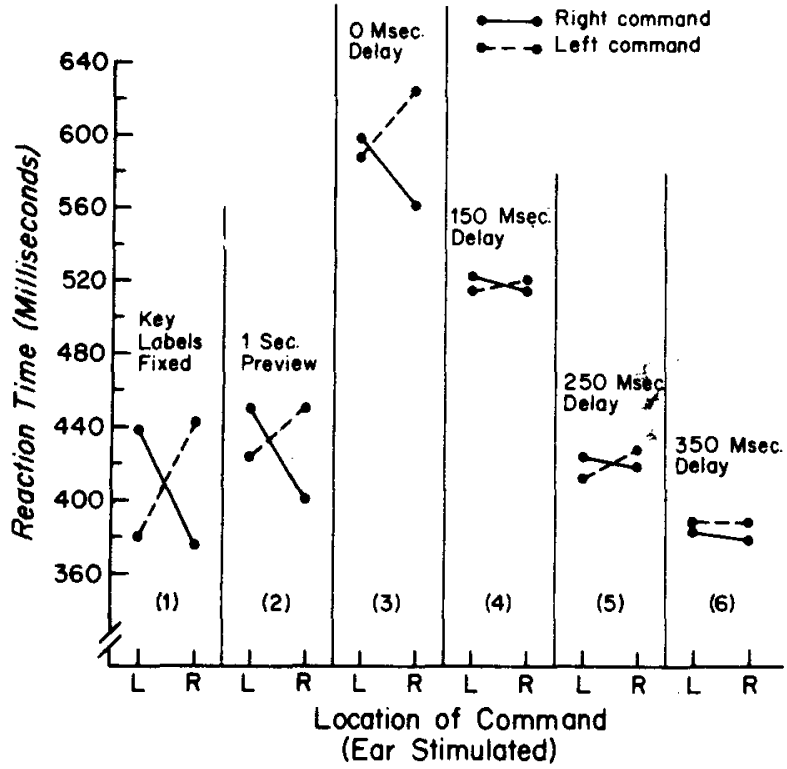

Figure 2. Effect of an irrelevant directional cue on the time required to translate pitch information into a keypress response. The basic task involved pressing either a left- or a right-hand key, labeled "red" or "green," in response to the pitch of a monaural tonal command. Each of six groups (see Panels 1-6) performed a different variation of this basic task. For Groap 1, the red key and the green key were labeled before the start of the experiment, and these designations remained fixed throughout. For Group 2, the keys were labeled $1 \mathrm{sec}$ prior to the onset of each tonal command and these labels varied from trial to trial; i.e., on one trial, the right key might be red and the left key green, while on the next trial the reverse labeling might occur. Reaction times for Groups 1 and 2 were measured from the onset of the tonal command. For Groups 3, 4, 5, and 6 , the key labels also varied from trial to trial. The labeling, however, occurred either simultaneously with presentation of the tonal command or 150,250 , or $350 \mathrm{msec}$ after the onset of the command. Reaction times for Groups 3-6 were measured from the instant the keys were labeled.

combinations in each of the six groups. An overall analysis of variance revealed a significant Ear Stimulated by Command by Groups interaction, $F(5,24)=7.97 . p<.01$, as well as an Ear Stimulated by Command interaction, $F(1,24)=60.43, p<.01$. and a main effect of groups, $F(5,24)=9.40, p<.01$.

Several follow-up analyses were then conducted to answer specitic questions. The performance of Group 1 was compared with that of Group 2 to examine the effect of changing the key labels from trial to trial. Note that in Group 1, the key labels were fixed throughout the experimental session, while in Group 2. the key labels varied over trials. Neither the groups main effect nor the Ear Stimulated by Command by Groups interaction, $F(1,8)=4.78$. $\mathrm{p}>.05$, were significant, indicating that performance was not affected by varying the key labels from trial to trial. The analysis did, however, reveal a significant Ear Stimulated by Command interaction, $F(1,8)=$ 64.07. $p>.01$, reflecting the effect of the irrelevant directional cue. 
To explore further the significant three-way Ear Stimulated by Command by Groups interaction in the overall analysis, separate analyses were conducted for Groups 3, 4. 5, and 6. Of primary importance was the finding that the Ear Stimulated by Command interaction was significant only for the 0 -msec delay (Group 3), $F(1,4)=8.98, p<.05$. In other words. the irrelevant directional cue had no effect on Groups 4,5 , and 6 , where the response keys were labeled after the onset of the tonal command.

In another analysis, comparing Group 3 with Group 2, there was a significant group effect, $F(1,8)$ $=39.35, \mathrm{p}<.01$, indicating that overall performance was faster when the keys were labeled prior to the tonal command than when both key labels and tonal command were presented simultaneously (430 vs. $592 \mathrm{msec}$ ). In this same analysis, the Ear Stimulated by Command interaction was significant, $F(1,8)=$ 20.66, $p<.01$, but the Ear Stimulated by Command by Groups interaction was not, $F<1$, suggesting that the effect of the irrelevant directional cue was identical for both groups.

An additional analysis was performed to determine whether the irrelevant directional cue associated with the ear stimulated had an inhibitory or a facilitative effect on information processing. For this analysis, the median RTs for each subject were averaged to form the following three categories of ear stimulated/command relationship: (a) a corresponding category. where the command was ipsilateral to the ear stimulated; (b) a noncorresponding category, where the command was contralateral to the ear stimulated; and (c) a binaural category, which provided an appropriate baseline since there is no directional cue associated with a command presented to both ears. Table 1 presents the means of each of these categories for each of the six treatment groups. An overall analysis of variance revealed a significant Categories by Groups interaction, $F(10,48)=6.07$, $\mathrm{p}<.01$, as well as a categories main effect, $F(2,48)=$ 40.24. $\mathrm{p}<.01$, and a groups main effect, $F(5,24)=$ 8.56, $\mathrm{p}<.01$.

Groups 1, 2, and 3 were then analyzed separately, since the previous analyses of the monaural treatments had shown that the effect of the irrelevant directional cue was restricted to these three groups. As expected, a significant categories main effect was found for each group. $F(2,8)=49.42, p<.01, F(2,8)$ $=10.67, \mathrm{p}<.01$, and $F(2.8)=9.63, \mathrm{p}<.01$, respectively. Follow-up Sheffé tests were then performed to determine whether the directional cue facilitated and interfered with performance for each group. For Group 1, corresponding responses were significantly faster than binaural baseline responses (378 vs. $409 \mathrm{msec}), F(2,8)=24.11, p<.05$, and binaural baseline responses were significantly faster

\begin{tabular}{|c|c|c|c|c|c|}
\hline \multirow[b]{3}{*}{ Group } & \multicolumn{5}{|c|}{$\begin{array}{r}\text { Time (in Milliseconds) to Tonal Commands as a } \\
\text { of Ear Stimulated/Command Relationship }\end{array}$} \\
\hline & \multirow[b]{2}{*}{ Treatment } & \multicolumn{4}{|c|}{$\begin{array}{l}\text { Ear Stimulated/Command } \\
\text { Relationship }\end{array}$} \\
\hline & & $\mathrm{C}$ & $\mathrm{NC}$ & B & Mean \\
\hline 1 & Key labels fixed & 378 & 440 & 409 & 409 \\
\hline 2 & 1-sec preview & 412 & 448 & 423 & 428 \\
\hline 3 & 0 -msec delay & 574 & 611 & 580 & 588 \\
\hline 4 & 150-msec delay & 514 & 520 & 527 & 520 \\
\hline 5 & 250-msec delay & 415 & 425 & 409 & 416 \\
\hline 6 & 350 -msec delay & 383 & 386 & 374 & 381 \\
\hline
\end{tabular}

Note. $C=$ corresponding, $N C=$ noncorresponding, and $B=$ binaural.

than noncorresponding responses ( 409 vs. $440 \mathrm{msec}$ ), $F(2.8)=25.31, p<.05$. These results indicate that when the key labels remained fixed throughout the block. the irrelevant directional cue facilitated performance on corresponding trials and interfered with performance on noncorresponding trials. Though Groups 2 and 3 showed a similar pattern to that observed in Group 1, i.e., baseline RT intermediate between corresponding and noncorresponding RT, in neither group was the facilitation effect statistically significant. However, for both Groups 2 and 3, there was a significant interference effect; i.e., noncorresponding trials were slower $(p<.05)$ than baseline trials. To summarize, then. both facilitation and interference occurred when the keys were labeled prior to the onset of the command and the labels remained constant throughout the session. Interference, but not facilitation, occurred when the key labels varied from trial to trial as iong as the labeling occurred prior to or simultaneously with the stimulus onset. The effect of the irrelevant directional cue disappeared entirely when the response keys were labeled after the tonat command was presented.

The mean RT data in Table 1 can be used to estimate the durations of various stages of information processing. The estimates, however, are complicated by the fact that a different group was involved in each treatment condition. The baseline treatment (Group 1) consisted of a typical two-choice $R T$ task in which the response keys were labeled before the start of the experiment and the labels remained fixed throughout. This condition yielded an average RT of $409 \mathrm{msec}$. There was a tendency for reactions to be slightly slower when the response keys were labeled $1 \mathrm{sec}$ prior to stimulus onset and the labels varied from trial to trial (Group 2). A marked increase in RT resulted when the key labels were designated simultaneously with stimulus onset (Group 3). This increase $(179 \mathrm{msec})$; reflects the additional time required to identify the lights and 
program the response. When the labeling of the response keys was delayed by 150,250 , or $350 \mathrm{msec}$, thus providing a longer and longer interval during which stimulus identification could proceed, RTs showed a substantial decline. However, again, as in Experiment I, subjects did not employ the interval to full advantage. In other words, the observed decrease in RT was less than the duration of the interval available for processing. For example, Group 4 was only $68 \mathrm{msec}$ faster than Group 3, despite the fact that they had an extra $150 \mathrm{msec}$ which might have been used for stimulus identification.

\section{Discussion}

Results of Experiment II indicated that the source of the tonal command affected information processing only when the response keys were labeled prior to or simultaneously with the onset of the tone. When the response keys were labeled after the onset of the tone, the irrelevant directional cue had no effect. This finding has important implications, because it suggests that the directional cue affects the response selection stage of information processing. The directional cue had an effect only when subjects could proceed promptly to the response selection stage. When the labeling of the response keys was delayed, subjects were prevented from proceeding to the response selection stage and so the directional cue in the stinulus tone had no effect.

The paradigm developed in Experiment Il may be of some general importance because it provides a means of separating response selection from the preceding stages of information processing. Smith (1968) points out that a complicating factor in theories of choice RT is the possibility of overlap between stages. He also notes that current theories have neglected response selection processes. We suggest that the present paradigm might prove useful for exploring a variety of problems related to durations of processing stages and the operations involved.

\section{GENERAL DISCUSSION}

Two different paradigms were employed to determine the duration of an interference effect associated with the source of a directional command. Previous research had shown that the eye or ear in which a command was presented provided an irrelevant directional cue which affected the time required to translate the symbolic content of the command into an appropriate keypress response. We had hypothesized that this effect was due to an initial tendency to react to the source of the stimulus rather than to its meaning and had argued that the slower information processing observed when source and content of a stimulus did not correspond was a result of the additional time required to override the initial incorrect response tendency. It seemed reasonable to expect that the interference would be short-lived and that it would dissipate if subjects were to delay their response momentarily. We therefore developed two tasks in which subjects did not respond immediately upon presentation of the stimulus but, instead, delayed their response for various specified intervals. By identifying the critical interval at which the interference phenomenon no longer occurred, we felt we could estimate the duration of the effect and also localize it in the information processing sequence.

In Experiment I, subjects saw a stimulus light but were told not to execute their response until they heard a go signal. Analysis of the findings at the various delay intervals indicated that the duration of the interference phenomenon was approximately 250 msec.

In Experiment II, subjects were asked to translate the pitch of a tone into an appropriate keypress response. For some groups, the stimulus-response connections were specified prior to or simultaneously with the presentation of the stimulus tone, while, for other groups, the labeling of the response keys followed the onset of the tone. Results indicated that the irrelevant directional cue affected performance only when the stimulus-response connections were specified prior to or simultaneously with the onset of the tone. In other words, when the task permitted subjects to proceed promptly to the response selection stage. the irrelevant cue had an effect; when the response selection stage was delayed, the irrelevant cue had no effect. Our findings then suggest that the directional cue affects the response selection stage of processing.

The present results might be better understood with reference to the following simple model for response selection. The model uses a computer program as an analogy to describe how response selection might be achieved. It is assumed that the subject establishes a response buffer for each required response in the experimental task; e.g.. a right and a left response-key buffer. Within each buffer is stored a list of the stimuli assigned to that response. After a stimulus has been identified, the subject's "central processing unit" (CPU) scans the response buffers looking for that stimulus. When the stimulus is located in a particular buffer, the response associated with that buffer is executed. It is further assumed that the source of the stimulus, i.e., left or right, serves to bias the subject toward scanning the corresponding response buffer first. Consequently, when the relevant content of the stimulus and its irrelevant directional component correspond, the correct response will be selected in the minimum time; when they do not correspond, response selection will be delayed due to the initial scanning of the wrong buffer. When there is no irrelevant directional cue, e.g., on binaural trials. RT should be of intermediate magnitude since there is 
no bias toward scanning a particular buffer first. Assuming random selection, the initial buffer selected should be correct on only half of the trials.

The above model accounts for the changes in the stereotype which were observed under the various conditions employed in Experiments I and II. For example, in Experiment I, under the 150-msec delay, the go signal occurred while the subjects were still identifying the stimulus, and so response selection proceeded in the same fashion as in the no-delay condition, i.e., RTs reflected a potent stereotypic reaction. However, when the go signal was delayed for more than $250 \mathrm{msec}$, the subject had time to scan both response buffers and select the appropriate response before the signal to execute was presented. Thus, under the long-delay conditions, RT no longer reflected the beneficial or detrimental effects produced by scanning a particular buffer first.

In Experiment II, the typical stereotypic reaction occurred when subjects were able to program the stimulus-response associations prior to stimulus presentation, i.e., when the response keys were labeled prior to the trial. The stereotype also occurred when the response keys were labeled simultaneously with the onset of the stimulus tone. Under this latter condition, subjects apparently first identified the key lights so they could load the response buffers. This additional step slowed RT but, once completed, subjects could proceed without delay through the stimulus identification and response selection stages. When labeling of the response keys was delayed, the sequence of operations was altered. Now, presumably, subjects first processed the tone due to its temporal priority and then proceeded to identify the key lights and load the buffers. If subjects had scanned the buffers immediately after tone identification, no response could have been selected since no information was contained in the buffers. Apparently, the engagement of the CPU in the process of identifying the lights and loading the buffers eliminated the initial scanning bias produced by the directional component of the tone and thereby eliminated the stereotype.

To summarize, the present research suggests that irrelevant directional cues produce the stereotypic reaction by affecting operations in the response selection stage of information processing. This conclusion was based on two findings: (a) results of Experiment I suggested that the effect occurred relatively late in the decision process, implicating the response selection stage, and (b) results of
Experiment II indicated that the stereotype did not occur when subjects could not proceed immediately to response selection after completing the stimulus identification stage.

\section{REFERENCES}

Craft, J. L.. \& Simon, J. R. Processing symbolic information from a visual display: Interference from an irrelevant directional cue. Journal of Experimental Psychology, 1970, 83, 415-420.

Fitss, P. M., \& Sefger, C. M. S-R compatibility: Spatial characteristics of stimulus and response codes. Journal of Experimental Psychology, 1953, 46. 199-210.

Jensen, A. R., \& Rohwer, W. D., JR. The Stroop color-word test: A review. Acta Psychologica, 1966, 25, 36-93.

Simon, J. R., Acosta, E., JR., \& Mewaldt, S. P. Effect of locus of warning tone on auditory choice reaction time. Memory \& Cognition, 1975, 3. 167-170.

Simon, J. R., \& Craft, J. L. Effects of an irrelevant auditory stimulus on visual choice reaction time. Joumal of Experimental Psychologv, 1970, 86, 272-274.

Simon, J. R., \& CRAFT, J. L. Reaction time in an oddity task: Responding to the "different" element in a three-light display. Journal of Experimental Psychology, 1972, 92, 405-411.

Simon, J. R.. Craft, J. L., \& Webster, J. B. Reaction time to onset and offset of lights and tones: Reactions toward the changed element in a two-element display. Joumal of Experimental Psychology, 1971, 89, 197-202.

Simon, J. R., CRaft, J. L., \& Webster, J. B. Reactions toward the stimulus source: Analysis of correct responses and errors over a five-day period. Journal of Experimental Psychology, 1973, 101, 175-178.

Simon, J. R., Small, A. M., Jr., Ziglar, R. A., \& Craft, J. L. Response interference in an information processing task: Sensory versus perceptual factors. Joumal of Experimental Psychology, 1970, 85, 311-314.

Smiтh, E. E. Choice reaction time: An analysis of the major theoretical positions. Psychological Bulletin, 1968, 69, 77-110.

WALLACE, R. J. S-R compatibility and the idea of a response code. Joumal of Experimental Psychology, 1971, 88, 354-360.

\section{NOTE}

1. Since the typical magnitude of the Command by Command Location interaction had been found to be on the order of $60 \mathrm{msec}$, it is difficult to conceive how the effect could persist undiminished for $250 \mathrm{msec}$ and yet have its onset early in the decision process. Initially, we had mistakenly believed that since the difference in RT associated with location of the command was about $60 \mathrm{msec}$, an interval of this duration would be sufficient to override the tendency to respond to the source of stimulation. Accordingly, two pilot experiments were conducted in which the intervals used were 0,30 , $60,500 \mathrm{msec}$ and $0,50,100,400 \mathrm{msec}$. Results from six subjects in each of these pilot studies showed that the effect of the irrelevant directional cue disappeared only at the longest intervals. i.e., 500 and $400 \mathrm{msec}$, respectively.

(Received for publication May 23, 1975; revision received August 27, 1975.) 